\title{
The Simulation and Test for Piezoelectric Composite Tube
}

\author{
WANG Hongwei \\ Beijing information science and technology university, Beijing 100192, China \\ drhwh@bistu.edu.cn
}

\begin{abstract}
Piezoelectric composite circular tube has been developed, this tube is used for underwater acoustic transducer. The finite element simulation has been done for piezoelectric composite material tube, and gained the tube structure parameters. In accordance with the structural parameters obtained from the simulation, the composite circular tube is processed and its performance has been tested. the results show as follow: Acoustic impedance is $18.05\left(\mathrm{~Pa} \cdot \mathrm{s} / \mathrm{m}^{3}\right)$, relative permittivity is 859 , sound velocity is $3200 \mathrm{~m} / \mathrm{s}, \mathrm{d} 33$ constant is $480 \mathrm{pC} / \mathrm{N}$, vibration displacement is $89.5 \mathrm{pm}$, the resonant frequency is $388 \mathrm{kHz}$, bandwidth is $11.2 \mathrm{kHz}$. The piezoelectric composite circular tubes suitable for horizontal omnidirectional broadband transducer.
\end{abstract}

Keywords: Piezoelectric Composite; tube; simulation; Properties

\section{Introduction}

Composite materials are multiphase materials, which are made of piezoelectric phases (such as PZT) and polymer phases. It overcomes the shortcomings of the single-phase piezoelectric material and retains the strong piezoelectric property of piezoelectric phase material with a lower Qm value. The bandwidth of the transducer element is inversely proportional to the Qm value of the material, so the piezoelectric composite material is especially suitable for making the wide band transducer[1-4]. The typical piezoelectric composite material is made by cutting the ceramic and casting polymer. This method makes planar array composites that the piezoelectric ceramic is cut into the ceramic column array and the flexible polymers (such as epoxy resin, rubber, etc.) are added in the ceramic column array .

Chen Jun bo et al. Of the No. 715 Research Institute of China Shipbuilding Heavy Industry Group made a piezoelectric composite piston transducer and the same size piezoelectric ceramic transducer [3]. The composite exhibits a single thickness resonance that the frequency is $180 \mathrm{kHz}$ and the $3 \mathrm{~dB}$ bandwidth is $15 \mathrm{kHz}$. However, the piezoelectric ceramic presents multi-peaks resonance and the maximum resonance bandwidth is only $5 \mathrm{kHz}$. The bandwidth of piezoelectric composite is 3 times that of single phase piezoelectric ceramic.

1-3-2 type piezoelectric composite broadband underwater acoustic transducer [4] was developed by Xian Xiao jun et al, who are in the institute of applied acoustics in Shanxi 
Normal University. The transducer is placed in anechoic test pool to test Water emission voltage response .The maximum response voltage of 1-3-2 type piezoelectric composite ceramic material underwater acoustic transducer is $183 \mathrm{~dB}$, and $-3 \mathrm{~dB}$ bandwidth of nearly $40 \mathrm{kHz}$.Its bandwidth is much larger than that of the ceramic.

Utilizing composite may stretcher transducer bandwidth, but it is limited by the preparation process of the composite material forming the only plane. So the bandwidth is limited. At present, most of the curved transducers use piezoelectric ceramics as the sensitive material, because its $Q$ value is much larger than that of the composite material. that is, the bandwidth of the material is less than that of the composite material. Therefore, if the composite material is made into a curved surface, the bandwidth of the transducer is bound to expand and the large open-angle beam radiation Sonic can be realized.

\section{Design and Simulation Composite Circular Tube}

The structure of 2-2 type Piezoelectric composite pipe shows in Figure 1.Composite pipe are made by cutting ceramic ring - pouring epoxy - Sample polish - coated electrode process. a piezoelectric ceramic pipe is cut by Accurate control of the knife gap and step to make composite ring arranged in a uniform array.

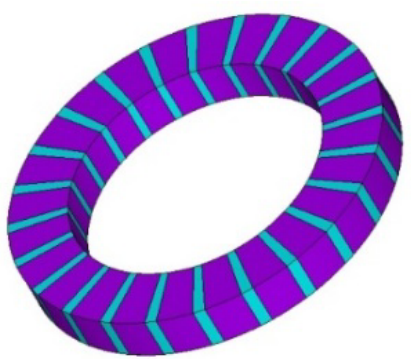

Fig.1 2-2 type Piezoelectric Composite pipe

The pipe structure parameters include the volume percentage of the composite piezoelectric ceramic $\mathrm{V}$, the radius of the circle $\mathrm{R}$, the thickness of the ring $\mathrm{T}$ and the height of $\mathrm{H}$. The volume percentage of the composite piezoelectric ceramic directly determines the electromechanical coupling coefficient $\mathrm{Kt}$ and Qm value of the composites.

In a cylindrical coordinate system modeling and simulation, it makes the piezoelectric ceramic that the height is $\mathrm{h}$, the inner radius is $\mathrm{r} 1$, the outer radius is $\mathrm{r} 2$ and the polar angle is from $0{ }^{\circ}$ to $8^{\circ}$. Then it makes the epoxy resin that the height is $\mathrm{h}$, the inner radius is $\mathrm{r} 1$, the outer radius is $\mathrm{r} 2$ and the polar angle is from $8{ }^{\circ}$ to $10^{\circ}$. The entity is copied 36 parts by the method of rotational symmetry to make the entire annular composite ring. The thickness of the composite ring is $4 \mathrm{~mm}$, The height of the composite ring is $3 \mathrm{~mm}$, the average radius of the composite ring is $17 \mathrm{~mm}$. By simulation, the resonance frequency is $372 \mathrm{KHz}$. 


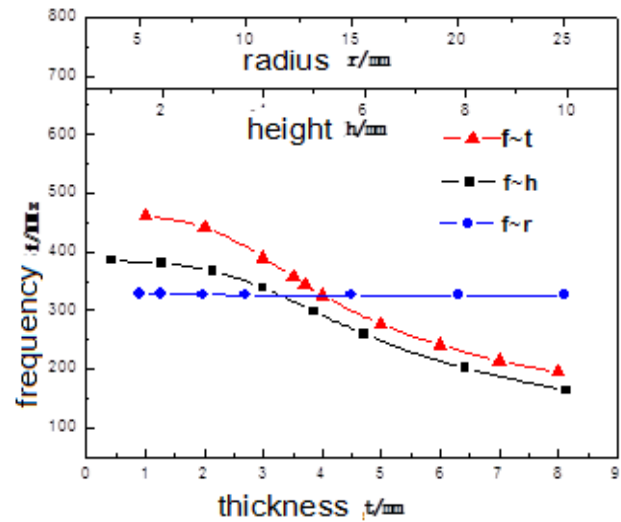

Fig.2The resonant frequency with the pipe parameters

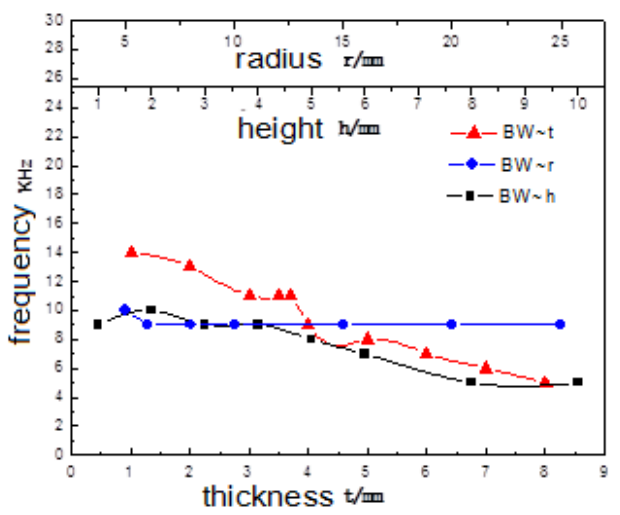

Fig.3The bandwidth of BW with the pipe parameters

From Figure2,3, we can know that the ring resonant frequency decreases with the increase of the ring thickness $t$ and the height $h$, and does not change with the change of the radius; The ring bandwidth BW also decreases with the increase of thickness $t$ and height $h$, and does not change with the change of radius.

By the above simulation data, the piezoelectric vibrator sensing element is determined. The ring height is $3 \mathrm{~mm}$, the inner radius is $13 \mathrm{~mm}$, the thickness is $4 \mathrm{~mm}$, the ceramic width is $1.6 \mathrm{~mm}$, the epoxy width is $0.4 \mathrm{~mm}$, and the unit width is $2 \mathrm{~mm}$.

\section{Preparation of Composite Pipe}

On the basis of the conventional cutting - casting process, Composite pipes are directly made by cutting ceramic ring - pouring epoxy - Sample polish - coated electrode process. Man continues to explore the preparation process .Especially a piezoelectric ceramic pipe is cut by Accurate control of the knife gap and step to make composite ring arranged in a uniform array.

the preparation and technical route of the composite pipe is shown in figure 4,5

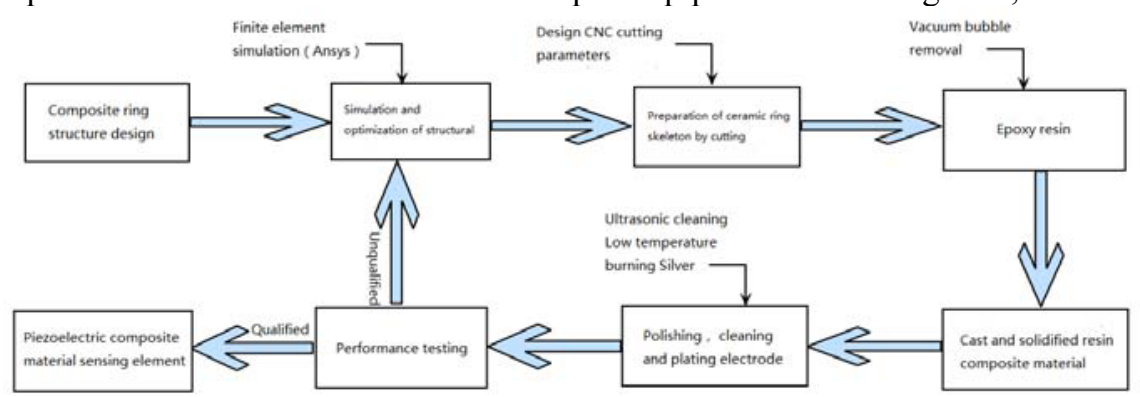

Fig.4 Preparation of composite circular tube array

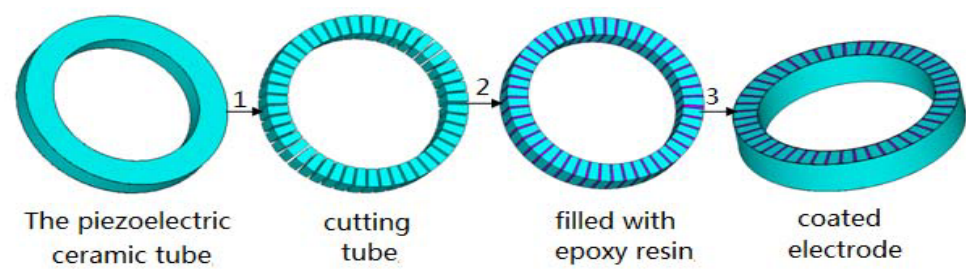

Fig.5 The preparation process of composite circular tubes 
The piezoelectric ceramic pipe is PZT-5 production produced by the acoustics Institute of Chinese Sciences Academy .The bottom surface of the piezoelectric ceramic pipe is placed on $1 \mathrm{~cm}$ thick square plates and is fixed with melted paraffin. After the paraffin solidification, the steel plate together with the ceramic pipe are placed into the cutting positions in the disco automatic grinding wheel dicing machine of the Japanese company (model DAD321). Then setting programs in the cutting machine and cutting the ring into 36 parts. Epoxy resins are produced fluid, and the epoxy resins are poured in the ceramic skeleton. At the same time, the bubble is evacuated and the epoxy resin is evenly filled. After Curing at room temperature, the composite material is obtained. Finally Polishing rough shaping makes a composite material pipe, and the inner and outer surface is plated electrode to make a piezoelectric composite ring.

Due to the preparation process of the polarization of the piezoelectric ceramic under normal temperature and atmospheric pressure, the effect of the electrode of the piezoelectric ceramic is little. So the polarization of the composite material is omitted. The composite material is made into a kind of ring as shown in figure 6 .

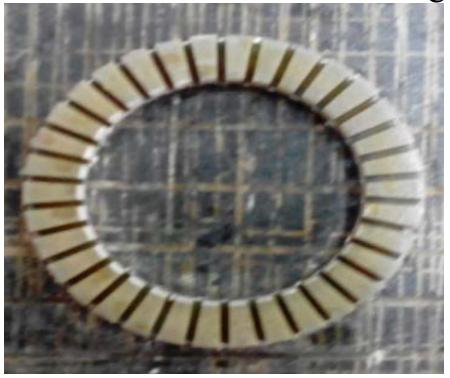

(a) cutting of PZT-5 piezoelectric ceramics

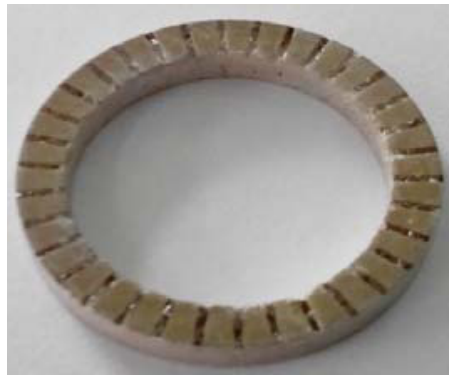

(b) Casting Epoxy resin, making electrode Fig.6 The preparation of composite circular tube array

\section{Performance Test of Composite Pipe}

The dielectric and piezoelectric properties of the composite pipe are tested. When the test frequency is $100 \mathrm{~Hz}$, the piezoelectric constant $\mathrm{d} 33$ of the piezoelectric composites is measured by the piezoelectric constant tester (model: ZJ-3D) ;Vibration displacement of composite materials was tested by laser scanning vibration instrument(Type: Polytec Scanning Vibrometer ); The low frequency capacitance and frequency characteristics of the composite material are tested by a precision impedance analyzer (Agilent 4294A).

Using the relationship is calculated as follows.

$$
\begin{aligned}
& \varepsilon_{r}=\frac{c t}{\varepsilon_{0} A} \\
& v=2 f_{p} t \\
& Z=\rho v
\end{aligned}
$$

$\mathrm{C}$ is the capacitance of the sample, $\mathrm{t}$ is the thickness, $\mathrm{A}$ is the surface area of the electrode, and $f_{\mathrm{p}}$ is the parallel resonance frequency. They can be directly measured. $\mathrm{v}$ is the acoustic wave propagation velocity in the sample, $Z$ is the acoustic impedance, and $\rho$ is the density .They can be get by computing .

The impedance characteristic of the piezoelectric composite pipe is shown in figure 7.The main vibration resonance of the composite material is much larger than other vibration, and 
the bandwidth is larger than that of the ceramic. These characteristics can improve the bandwidth and frequency selectivity of the transducer, so the design of the transducer array is easy to implement.

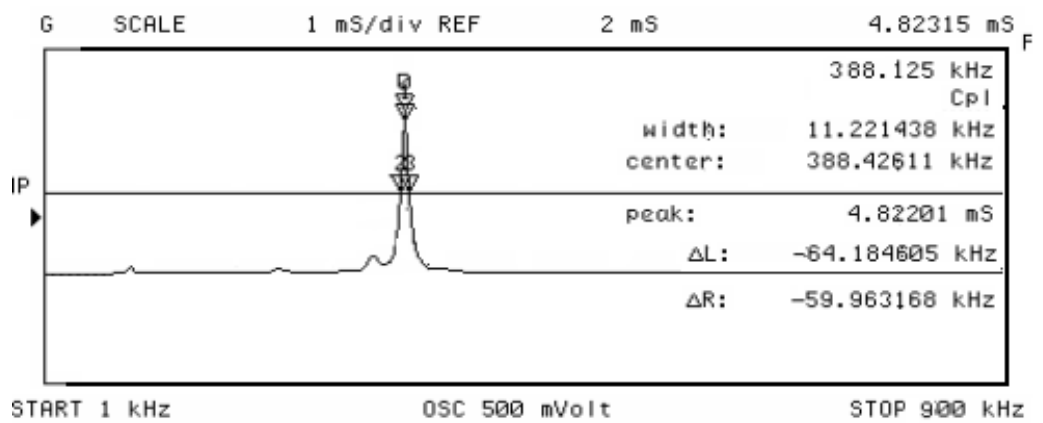

Fig.7 Impedance characteristic curve of the ring of 2-2 type piezoelectric composite material

The sample parameters were measured, such as Piezoelectric constant, the vibration displacement ( $5 \mathrm{~V}$ voltage drive), the relative dielectric constant, the speed of sound, acoustic impedance and so on. As is shown in table 1.

Table 1 Properties Of Composite

\begin{tabular}{|c|c|c|c|c|c|c|c|c|c|c|}
\hline Material & $d_{\mathrm{N})}^{d_{33}}(\mathrm{pC}$ & $\begin{array}{l}\text { Vibration } \\
\text { displacem } \\
\text { ent (pm) }\end{array}$ & $\varepsilon_{\mathrm{r}}$ & $\mid \begin{array}{l}\text { Dielec } \\
\text { tric } \\
\text { loss }\end{array}$ & $\begin{array}{l}f_{\mathrm{s}} \\
(\mathrm{kH} \\
\mathrm{z})\end{array}$ & $\begin{array}{l}f_{\mathrm{p}} \\
(\mathrm{kH} \\
\mathrm{z})\end{array}$ & $\begin{array}{l}\mathrm{B} \\
\mathrm{W} \\
(\mathrm{kH} \\
\mathrm{z})\end{array}$ & $\begin{array}{l}v \\
(\mathrm{~m} / \mathrm{s} \\
)\end{array}$ & $\begin{array}{l}\rho \\
(\mathrm{kg} / \mathrm{m} \\
\left.{ }^{3}\right)\end{array}$ & $\begin{array}{l}Z \\
\text { (Mraly) }\end{array}$ \\
\hline $\begin{array}{l}\text { piezoelect } \\
\text { ric } \\
\text { composite } \\
\text { S }\end{array}$ & 480 & 89.5 & 859 & 0.04 & 388 & 489 & $\begin{array}{l}11 . \\
2\end{array}$ & $\begin{array}{l}320 \\
0\end{array}$ & $\begin{array}{l}5018 . \\
7\end{array}$ & 18.05 \\
\hline
\end{tabular}

The test and simulation results are compared. The test resonant frequency $\mathrm{f}$ is $388 \mathrm{KHz}$ and the bandwidth BW is $11.2 \mathrm{kHz}$. The simulation resonance frequency $\mathrm{f}$ is $372 \mathrm{kHz}$ and the bandwidth $\mathrm{BW}$ is $9 \mathrm{kHz}$. The highest frequency difference is $4.3 \%$, and the bandwidth difference is $24.4 \%$.

\section{Summary}

The piezoelectric composites is made by cutting - casting technique . By test, the resonant frequency is $388 \mathrm{KHz}$, the bandwidth is $11.2 \mathrm{kHz}$, the material piezoelectric constant $\mathrm{d} 33$ is $480 \mathrm{pC} / \mathrm{N}$, the vibration displacement is $89.5 \mathrm{pm}$, the sound speed is $3200 \mathrm{~m} / \mathrm{s}$, the Acoustic impedance is $18.05 \mathrm{Mraly}$, and the relative dielectric constant is 859 .The piezoelectric constant $\mathrm{d} 33$ and the vibration displacement of the 2-2 type composite can reach the performance of the piezoelectric ceramics, and the sound speed and the acoustic impedance are greatly reduced, the bandwidth is increased.

At present, the composite molding process is limited to curved surface. Therefore This not only limits the transducer beam, but also restricts the further development of transducer. In this paper, Composite pipe array is directly made by the process of cutting ceramic ring pouring epoxy - sample grinding - curved screen coated electrode.

If you want to further broaden the bandwidth, it can be two different frequencies of single-tube composite pipes stacked to produce a coupling that can further broaden the Bandwidth. 


\section{Acknowledgements}

1. The paper is supported by two fund projects:

2. Project of National Natural Science Foundation of China (614710470);

3. Graduate School of Beijing Information Science and Technology University project (5111523902);

\section{References}

1. Liu Huisheng, MO Kihei, longitudinal study design broadband transducer progress, acoustic technology, Vol. 33 No. 6, December 2014, p564-571.

2. Christopher J, Purcell A, Richard F. Multi-mode pipe projector: United States, 6584039[P].2003-06-24.

3. Li Li.1 -3 type of piezoelectric composite materials and underwater acoustic transducer research. Beijing University of Posts and Telecommunications doctoral dissertation.May 2008.

4. Bin Zhang, Likun Wang, Lei Qin etc. Influence of Volume Fraction of Piezoelectric Ceramics on Popertiesof PZT672/Epoxy1-3 Piezocomposite. Advanced Materials Research, Vols. 989 (2014) p 364-368.

5. Chen Junbo, Wang Yuebing,Zhong Lin Jian. 1-3 type piezoelectric composite and ordinary PZT transducer performance contrast analysis. Acoustic and electronic engineering, 2007 (3):p25-27

6. Xian Xiaojun,Lin Shuyu, Deng pan Wang, Ma chin Yi.Based on 1-3-2 type piezoelectric composite electric broadband acoustic transducer, piezoelectric and acoustooptic, Vol 36 No. 4, August 2014.P491-497. 